\title{
Colaboración con empresas en la docencia de asignaturas en el marco del ABP \\ Collaboration with companies in the teaching of subjects in the framework of PBL
}

\author{
Eduardo Manchado Pérez ${ }^{1}$, Carlos Romero Piqueras ${ }^{1}$, José María López Pérez ${ }^{2}$, Roberto Casas Nebra ${ }^{2}$ \\ manchado@unizar.es, carlos.romero@unizar.es,chlopez@unizar.es,rcasas@unizar.es \\ ${ }^{1}$ Departamento de Ingeniería de Diseño y \\ Fabricación \\ EINA, Universidad de Zaragoza \\ Zaragoza, España \\ ${ }^{2}$ Departamento de Ingeniería Electrónica y \\ Comunicaciones \\ EINA, Universidad de Zaragoza \\ Zaragoza, España
}

\begin{abstract}
Resumen- El aprendizaje basado en proyectos se ha revelado en los últimos años como una herramienta de gran utilidad para obtener excelentes resultados en cuanto a la adquisición de competencias formativas, especialmente en cuanto a la consideración del ratio esfuerzo/logro. En este sentido una de las claves de su éxito radica en la capacidad de emular del modo más realista posible la actividad profesional dentro del ámbito empresarial, por lo que la participación de compañías en el espacio docente, propiciando el desarrollo de proyectos reales bajo encargo, representa una interesante oportunidad. En esta presentación se expone la experiencia y las características de la incorporación de una empresa de gran prestigio al entorno docente de los Grados de Ingeniería en Diseño Industrial y Desarrollo de Producto y del Grado en Ingeniería Electrónica y Automática de la Escuela de Ingeniería y Arquitectura de la Universidad de Zaragoza.
\end{abstract}

Palabras clave: ABP, empresa, interdisciplinar, diseño, electrónica, ingeniería.

Abstract- Project-based learning has emerged in recent years as a very useful tool for obtaining excellent results in terms of acquiring training skills, and especially in terms of the effort / achievement ratio. In this sense, one of the keys to its success lies in the ability to emulate in the most realistic possible way the professional activity within the business environment, so that the participation of companies in the teaching space, favoring the development of real projects under commission, represents an interesting opportunity. This presentation shows the experience and the characteristics of the incorporation of a company of great prestige to the teaching environment of the Degrees of Engineering in Industrial Design and Product Development and of Electronic and Automatic Engineering of the School of Engineering and Architecture of The University of Zaragoza.

Keywords: PBL, companies, interdisciplinar, design, electronics, engineering.

\section{INTRODUCCIÓN}

El aprendizaje basado en proyectos (ABP) se ha convertido en una estrategia ampliamente aceptada en los últimos tiempos como un recurso que permite articular la docencia universitaria en múltiples áreas de conocimiento (como son las ingenierías), debido a su probada eficacia en diversos aspectos:

- Orienta los contenidos teóricos hacia su aplicación práctica, contribuyendo a su puesta en valor.

- Se fundamenta en la adquisición de conocimientos por medio de la experiencia, lo que contribuye a fijar conceptos de un modo más eficaz.

- $\quad$ Bien gestionado, resulta altamente motivador para los estudiantes, que visualizan los posibles logros profesionales que podrán obtener gracias al esfuerzo realizado en la época de aprendizaje.

- Contribuye al desarrollo y puesta en práctica de competencias transversales como el trabajo en equipo, la autogestión, el desarrollo de diferentes roles, o la asunción de responsabilidades, entre otros.

Desde la experiencia en el desarrollo continuado y exitoso de programas docentes basados en $\mathrm{ABP}$ en la Escuela de Ingeniería y Arquitectura (EINA) de la Universidad de Zaragoza, es posible afirmar que una clave de su éxito radica en el hecho de que la experiencia sea capaz de replicar del modo más fidedigno posible las condiciones de trabajo de un contexto profesional real, tal como ha sido reconocido en diferentes ocasiones, (Blanco et al, 2015. Manchado et al, 2015).

En este sentido, el Grado de Ingeniería en Diseño Industrial y Desarrollo de Producto de la EINA - UZ ha puesto en práctica de modo pionero diversas prácticas que han probado su éxito, obteniendo altas tasas de éxito y rendimiento y de satisfacción de egresados y empleadores, como son los trabajos de módulo de asignaturas (Manchado Pérez, E., \& López Forniés, I., 2012). No obstante, la actividad más ambiciosa en cuanto a su capacidad de replicar el contexto profesional real, permitir el desarrollo de competencias transversales y motivar a los estudiantes es la de desarrollo de "proyectos híbridos", implementada junto al Grado de Ingeniería Electrónica y Automática (López Pérez et al, 2013). En esta actividad se produce una colaboración intergrados en la que los estudiantes trabajan en equipos multidisciplinares y 
en el contexto de diferentes asignaturas para desarrollar un proyecto de producto en el que deben asumir responsabilidades, programar tareas, y conseguir el entendimiento entre personas procedentes de diferentes disciplinas para obtener un éxito compartido.

En esta aportación se expondrá cómo esa actividad ha dado un paso más en sus últimas ediciones, incorporando de manera eficaz la presencia de empresas a la actividad docente, las dificultades que ha sido necesario sortear para conseguir ese objetivo y cómo se ha conseguido obtener valiosos resultados.

\section{CONTEXTO}

La presencia de las empresas y su participación en el ámbito universitario es un aspecto altamente relevante sobre el que existen no pocos puntos de discusión:

\section{A. Motivación de la colaboración.}

Es posible encontrar enfoques muy diferentes a la hora de analizar las posibles razones que motivarían la inclusión de empresas y su grado de implicación en el ámbito universitario. Existen posturas que van desde el convencimiento de que los contenidos de los programas docentes deberían estar orientados a la satisfacción de las necesidades de las empresas que en el futuro emplearán a los profesionales formados (la universidad es así un recurso social cuya finalidad es proveer al tejido productivo, económico y cultural, de los profesionales demandados), hasta el convencimiento de que son las empresas las que están en deuda con la universidad y deberían contribuir decididamente a su financiación o mecenazgo a fondo perdido (desde la perspectiva de su responsabilidad social corporativa, la universidad adoptaría un rol de liderazgo prospectivo respecto de la definición de los roles profesionales que interesan al conjunto de la sociedad, y esa tarea sería recompensada por el tejido productivo, económico y cultural, que se beneficia en última instancia de la misma).

\section{B. Responsabilidad pública/privada.}

La idoneidad del carácter público/privado de la institución universitaria es otra cuestión de discusión; por un lado se argumenta que el carácter universal e independiente que debería tener la institución universitaria sólo se puede garantizar si su gestión y financiación se desarrollan desde el ámbito de las administraciones públicas, mientras por otro lado hay quien opina que dicho modelo es insostenible a medio plazo y las universidades deben obtener canales de financiación privados (como ocurre, por ejemplo, en el ámbito anglosajón).

\section{Diferentes modelos organizativos.}

El modo en que se organiza la actividad de una institución universitaria difiere en muchos aspectos de los modelos habituales en el ámbito empresarial: existen diferentes horarios, diferentes calendarios, hay dificultades de programación derivadas de la necesidad de encajar y coordinar actividades dentro de encorsetados planes semestrales, las estructuras jerárquicas presentan diferentes formatos, y los procedimientos administrativos son muy diferentes, dificultando la colaboración.

\section{Procesos de control y garantías.}

Desde la perspectiva empresarial, el modelo de relación con los estudiantes, de los que la universidad es responsable, no siempre se comprende bien; en ocasiones se tiende a confundir a los estudiantes con empleados, cuando la realidad es que son más bien clientes. Razón por la que la institución universitaria se debe a ellos, teniendo la obligación de preservar un rango de derechos, y establecer una serie de mecanismos de control y garantías (por ejemplo, en lo concerniente a los procesos de evaluación) que las empresas perciben en ocasiones como trabas burocráticas.

Para tratar de resolver esta necesaria relación de un modo satisfactorio, la Universidad de Zaragoza, dispone, como la mayoría de instituciones universitarias, de diferentes modelos de colaboración institucional con empresas, algunas de cuyas características son las siguientes:

- Prácticas universitarias: Encajadas o no como parte del currículo, se gestionan como una estancia complementaria del estudiante en la empresa, desarrollando actividades típicas de un empleado de la misma, aunque bajo supervisión docente.

- Desarrollo de trabajos de fin de estudios: Las empresas tienen la oportunidad de proponer y acordar con PDI de la Universidad temas de desarrollo e investigación que son acometidos por los estudiantes contando con una dirección docente que puede ser compartida con personal de la empresa.

- Contratos de investigación y desarrollo: Acordados ad hoc, gestionados por organismos responsables de la transferencia de resultados como es la OTRI de Zaragoza, prácticamente consisten en un modelo de prestación de servicios desde la estructura universitaria a demanda de las empresas con intereses puntuales.

- Cátedras Universitarias: Habitualmente consideradas como modelos de mecenazgo o de contribución solidaria desde la óptica de la responsabilidad social corporativa de determinadas empresas, desempeñan todo tipo de actividades de interés común.

\section{DESCRIPCIÓN}

En el caso de la actividad "Proyectos Híbridos”, estudiantes de las asignaturas "Laboratorio de diseño electrónico" y "Oficina de proyectos” del Grado de Ingeniería en Electrónica y Automática y "Metodología de Diseño” y “Oficina Técnica” del Grado de Ingeniería en Diseño Industrial y Desarrollo de Producto se organizan en equipos interdisciplinares para acometer el desarrollo de proyectos desde el convencimiento de que el ABP, y especialmente en el ámbito de las enseñanzas técnicas, es el método más eficaz para la adquisición de competencias específicas y transversales, la obtención de adecuados resultados de aprendizaje y el mejor valor en términos del ratio esfuerzo/logro.

Esencialmente los alumnos de las diferentes especialidades reciben su correspondiente formación teórica que posteriormente deben aplicar en el contexto de un proyecto conjunto, que desarrollan de modo colaborativo, bajo la supervisión coordinada de un equipo de profesores de las diferentes asignaturas. Los profesores colaboran puntualmente en la docencia de las asignaturas del otro Grado, y se 
programan una serie de actividades conjuntas en las que participa todo el grupo, apoyándose para ello en diferentes metodologías de trabajo; la experiencia cuenta con una trayectoria de años en los que no ha parado de crecer, sorteándose diferentes dificultades y obteniendo valiosos resultados (Manchado et al, 2015).

En este contexto parece natural que, buscando la mayor aproximación posible a la reproducción de un contexto real de trabajo, se haya perseguido la incorporación de una empresa a la experiencia, y que dicha incorporación se haya analizado y considerado desde la perspectiva docente, propiciando su evolución y ajuste al proyecto ya consolidado.

\section{A. Origen de la colaboración.}

Independientemente de los modelos de colaboración con empresa referidos anteriormente, o de que en los diferentes Grados se trate de fomentar la presencia de profesionales en charlas o clases magistrales (como las que articula el programa Expertia), en el Grado de Ingeniería en Diseño Industrial y Desarrollo de Producto ya existía una experiencia anterior de participación de empresas en las asignaturas. Esta participación, generalmente basada en acuerdos informales, consistía esencialmente en que diferentes empresas (con las que en ocasiones existían contactos profesionales previos, o con las que se habían desarrollado contratos de investigación o trabajos de fin de estudios), proponían un tema para el desarrollo de algún proyecto en las asignaturas, facilitando además una visita a sus instalaciones y quizá una sesión de valoración de los desarrollos de los alumnos (Remolques Beguer, Dynamobel, entre otros). Cuando las empresas tenían interés en acordar algún tipo de restricción sobre compromisos de confidencialidad, o de ejercer algún tipo de derecho sobre los resultados (Araven, Imaginarium, Pikolín, entre otros), el proceso se complicaba mucho ya que ninguno de los modelos existentes de colaboración prevé este tipo de relación, siendo necesario adaptar alguno de los mismos, lo que, dada la estructura jerárquica de responsabilidades y toma de decisiones en la institución universitaria podía retrasar enormemente todo el proceso.

Como consecuencia, por un lado, de esta experiencia y, por otro, del alto nivel de presencia e implicación de la empresa $\mathrm{B} / \mathrm{S} / \mathrm{H} /$ (principalmente por medio de la Cátedra B/S/H/ Unizar) en el contexto del Grado en Ingeniería Electrónica y Automática, se planteó en el curso 2014/15 el desarrollo de una experiencia piloto para la actividad de "proyectos híbridos”, similar a la realizada anteriormente con otras empresas en el Grado de Ingeniería de Diseño Industrial y Desarrollo de Producto. Así, la principal aportación en este caso era la presencia de una empresa de este nivel de reconocimiento, cuyo compromiso con la Universidad de Zaragoza y nivel de estrecha colaboración es muy destacado, siendo beneficioso para ambas partes. Era la primera vez además que se desarrollaban proyectos en el contexto de asignaturas, en un formato intergrados y con una participación tan numerosa de estudiantes y resultados (en torno a 17 proyectos y 70 estudiantes de ambas titulaciones).

\section{B. Revisión de los primeros resultados y obtención de un acuerdo estable.}

Por iniciativa de miembros responsables de la Cátedra, los resultados del proyecto fueron revisados en una serie de reuniones conjuntas entre responsables de desarrollo de producto de la empresa, y los profesores participantes. En dichas reuniones se tuvo en consideración el resultado de aprendizaje obtenido en el ámbito docente, el resultado de potencial transferencia de conocimiento (e incluso de posible obtención de patentes), y los beneficios institucionales de carácter general en términos de imagen y reputación corporativa. Parte de los resultados fueron mostrados en la exposición "proyectos emergentes" del edificio Paraninfo, contando con una gran presencia institucional por parte de la empresa.

Como conclusión a dicho análisis, se adoptaron los siguientes acuerdos:

- Firma de un convenio anual para el desarrollo del proyecto, en forma de contrato de investigación y desarrollo gestionado por OTRI, entre responsables de la empresa y representantes de la universidad de Zaragoza y del equipo docente.

- $\quad$ Acuerdo de programación de posibles temas de trabajo para los proyectos a desarrollar.

- Realización de visitas a las instalaciones de la empresa, por parte de los estudiantes y profesores.

- Participación de personal de la empresa en sesiones de seguimiento y valoración del trabajo de los estudiantes.

- Firma de acuerdos de confidencialidad y posible transferencia de resultados entre universidad, empresa y participantes.

- Asignación de una dotación económica específica que permita el mejor desarrollo de las prácticas de las asignaturas, a gestionar directamente por los profesores responsables. (Bajo estas premisas se desarrolló la actividad del curso 2015/16 y 2016/17).

- Adecuación del Premio a la innovación en el marco de la Cátedra B/S/H/, orientado al tema de trabajo propuesto cada año para los “proyectos híbridos”. los responsables de la empresa se implican así metodológicamente, contribuyendo a fijar objetivos y criterios del ABP.

\section{Consolidación de la actividad.}

En el curso 2016/17 la actividad se ha desarrollado de nuevo en términos similares, si bien se ha contado con algo más de financiación y con un desarrollo más fluido al contarse con la experiencia anterior. Al final del curso se ha realizado una valoración de los resultados, cuyas conclusiones han sido la conveniencia de avanzar en el establecimiento de la colaboración como una entidad independiente, y articulada en forma de renovación cíclica curso a curso que, no obstante, deba encontrar acomodo entre la estrategia y programación anual de actividades de desarrollo conceptual de producto de la propia empresa. Es decir, desde la empresa se tiende ya a considerarla como una actividad estratégica propia.

La valoración por parte de la empresa está en consonancia con su implicación y barre varios aspectos, según sus propios testimonios. Por una parte constatan el interés que ha despertado dentro y fuera de la empresa (incluyendo la dirección de la empresa en Alemania) así como el suscitado en los propios estudiantes hacia la empresa. De hecho se ha visto aumentar notablemente las solicitudes de prácticas. Por otra parte, desde la empresa se han visto gratamente sorprendidos 
por la calidad de los proyectos y el potencial de innovación para $\mathrm{B} / \mathrm{S} / \mathrm{H} /$ presente en los productos.

\section{RESUltados}

En estos tres cursos ha resultado posible evaluar el impacto e interés de la experiencia mediante los siguientes recursos:

- Reuniones de seguimiento y análisis del equipo docente.

- Reuniones de seguimiento y análisis con los responsables de la Cátedra y las áreas de Desarrollo en la empresa.

- Encuestas oficiales de satisfacción de los estudiantes participantes.

- Otras encuestas de valoración realizadas a estudiantes.

- $\quad$ Resultados de transferencia de la investigación.

Como resumen de los resultados obtenidos, se podrían destacar los siguientes:

La experiencia resulta muy valiosa para los estudiantes participantes, quienes se ven muy motivados por el hecho de intervenir en proyectos procedentes de un encargo real, algo para lo que es fundamental que los responsables del proyecto en la empresa hayan estado presentes en el entorno docente mostrando un alto grado de interés e implicación.

La disposición de recursos económicos que aplicar en el contexto de las prácticas facilita el alcance de mejores logros, lo que redunda en los resultados académicos y en la valoración de los estudiantes (ya que, por ejemplo, disponen de material para ejecutar prototipos funcionales).

Los proyectos desarrollados obtienen diferentes tipos de evaluación, lo que tiene un potencial interesante aunque no siempre es bien comprendido por los estudiantes; así, obtienen una evaluación docente centrada principalmente en la obtención de los resultados del aprendizaje, lo que no debe estar necesariamente ligado al resultado del proyecto propiamente dicho, mientras que la empresa puede acabar seleccionando un proyecto y acordando una compensación económica con sus autores porque, independientemente de lo que hayan aprendido, han obtenido un resultado patentable. Cabe resaltar que es éste precisamente uno de los puntos que más se trabajó en el convenio de tal modo que se consiguiera un equilibrio entre los intereses de la empresa (con gran experiencia en la protección de resultados) y los de los alumnos (con nula experiencia en el tema).

Por último, puede darse el caso de que un equipo (y su proyecto) obtenga una tercera evaluación discrepante dentro del contexto de los premios de la Cátedra B/S/H a los que pueden optar, ya que el criterio en este caso es principalmente el grado de innovación y prospectiva.

\section{CONCLUSIONES}

La actividad se ha consolidado y es claramente sostenible. Por este motivo es por lo que se ha considerado oportuno presentar este trabajo en el foro de CINAIC, ya que se entiende que puede servir de aporte para promover iniciativas similares en otros Grados.

Es necesario, no obstante, reclamar de los responsables de la gestión universitaria un esfuerzo para propiciar marcos formales que faciliten desde un punto de vista administrativo la gestión de este tipo de colaboraciones de un modo más ágil y eficaz. Los plazos y pasos de ratificación de los acuerdos, de gestión de gastos, de transferencia de resultados, así como determinados aspectos legales y administrativos (confidencialidad, encaje con los diferentes modelos de evaluación a que tienen derecho los estudiantes, entre otros puntos) ponen en peligro la disponibilidad de las empresas a participar en estas iniciativas y exigen de un esfuerzo personal por parte de los profesores que restan la motivación necesaria para su ejecución.

Hay que destacar que al integrar varias asignaturas y, sobre todo, de distintos grados, las dificultades de coordinación docente en cuanto a plazos y exigencias de trabajo de cada asignatura sean cada curso un reto difícil de superar, sobre todo porque tiene que encajar con el desarrollo físico de los prototipos. El que estas experiencias intergrados estuvieran contempladas en los planes de estudios a nivel de centro, de manera que se plantee la coodinación desde un nivel superior, facilitaría enormemente su desarrollo.

\section{AgRADECIMIENTOS}

A los estudiantes participantes en la experiencia por su entusiasmo y energía, a los responsables del proyecto en la empresa y Cátedra B/S/H por su interés e implicación, y al equipo directivo en la EINA por su apoyo a esta iniciativa.

\section{REFERENCIAS}

Blanco, T., Casas, R., Manchado-Pérez, E., Asensio, Á., \& López-Pérez, J. M. (2015). From the islands of knowledge to a shared understanding: interdisciplinarity and technology literacy for innovation in smart electronic product design. International Journal of Technology and Design Education, 1-34.

López Pérez, J.M, Manchado Pérez, E., Casas Nebra, R., López Forniés, I., Blanco Bascuas, T. (2013). Adquisición de competencias profesionales mediante proyectos interdisciplinares. Aprendizaje, Innovación y Competitividad. Actas del Congreso CINAIC 2013. 1, pp. 385 - 390. 2013. ISBN 9788469589274

Manchado Pérez, E., \& López Forniés, I.(2012). Coordinación por módulos de asignaturas en el Grado de Ingeniería de Diseño Industrial y Desarrollo de Producto de la Universidad de Zaragoza. Revista de Docencia Universitaria, 10(3).

Manchado Pérez, E., Romero Piqueras, C., Blanco Bascuas, T., Casas Nebra, R., López Pérez, J.M. (2015). Una experiencia de aprendizaje colaborativo, basada en la adaptación de metodologías de Design Thinking. La sociedad del aprendizaje. Actas del iii congreso internacional sobre aprendizaje, innovación $y$ competitividad. Cinaic 2015. 1, pp. 434 - 439. 2015. Isbn 9788460829072 